\section{Low Vacuum SEMs: Latest Generation Technologies and Applications}

\author{
William Neijssen, Ben Lich, \& Pete Carleson* \\ FEI Company, Eindhoven, The Netherlands \\ ${ }^{\star}$ FEI Company, Hillsboro, OR, USA \\ William.neijssen@fei.com
}

\section{Introduction}

Since becoming popular more than a decade ago, low vacuum scanning electron microscopes (SEM) have continued to evolve. The latest systems offer uncompromised performance over an unprecedented range of sample chamber vacuum conditions. Instruments are now available that provide near-nanometer resolution in all vacuum modes and the ability to operate at pressures as high as 4000 Pascals ( 30 Torr). Low vacuum operation eliminates much of the sample preparation required for conventional (high vacuum) SEM. Insulating samples can be imaged without conductive coatings. Wet, dirty, outgassing samples can be examined without drying and fixing. Systems can also be configured with a wide range of ancillary capabilities for imaging, analysis, and sample manipulation, including advanced secondary, backscattered,
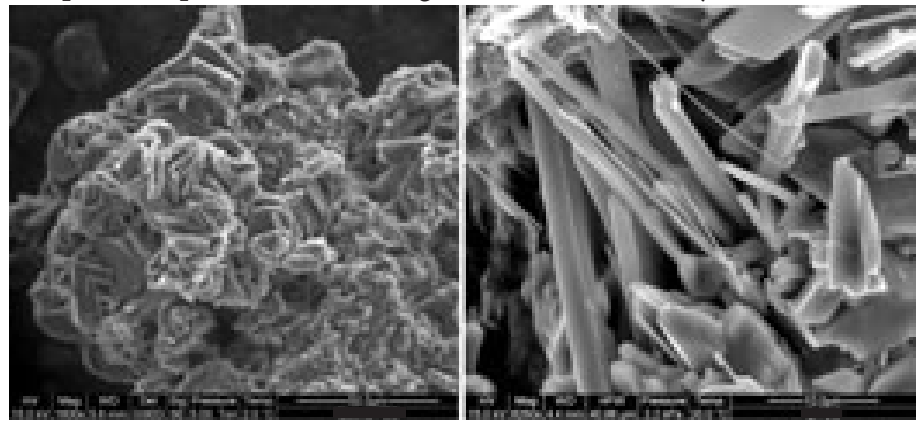

Fig 1 Hydrating $\mathrm{FeSO}_{4}$-ESEM mode supports chamber pressures sufficient to maintain water in the liquid state. This image was part of a hydration study observing the behavior of $\mathrm{FeSO}_{4}$ particles as water condensed on the cooled sample in the vapor filled chamber.

Fig 2 Hydrated Friedland gips - This gips sample is fully hydrated at 2200 Pascals and $20^{\circ} \mathrm{C}$. Water is an important structural component in many samples. ESEM can maintain such samples in a fully hydrated state indefinitely, permitting extended observations without dehydration induced changes in morphology.
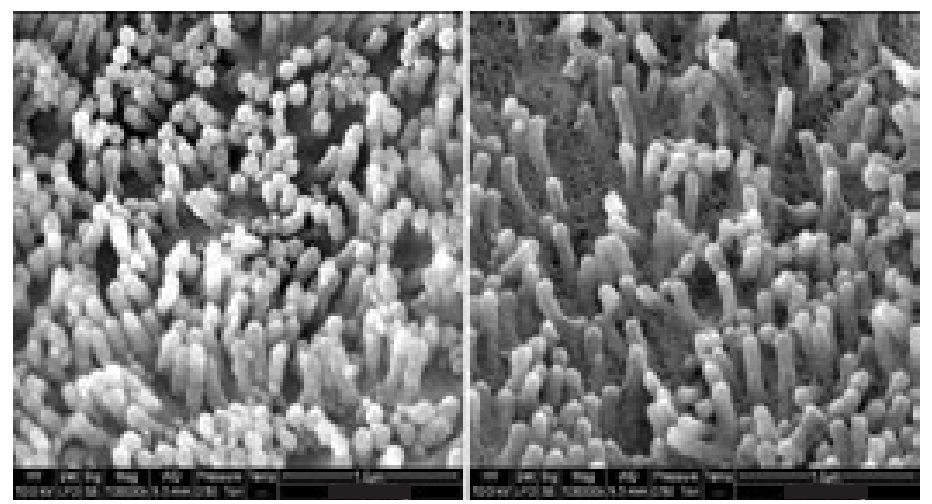

Fig 3 Gold Coated Tissue Samples - Conductive coatings are not always enough to neutralize charge, especially on samples that have extreme surface topography. Both of these images are gold coated tissue samples. The left image, acquired in high vacuum mode, shows charging effects and was quite unstable. In the right image, taken in low vacuum mode, the charging has disappeared and the image was quite stable.

SEM developed initially as a high-vacuum technique, but has been extended in recent years to permit observations throughout a range of vacuum conditions. That range is conveniently divided into three segments based on the maximum pressure permitted in the sample chamber: high vacuum $<10^{-2} \mathrm{~Pa}$, low vacuum $<500 \mathrm{~Pa}$, and extended low vacuum $<4000 \mathrm{~Pa}$.

High-Vacuum SEM - High vacuum is required throughout the electron columns of all SEMs to prevent scattering as electrons travel from the source through the beam forming lenses to the specimen in the sample chamber. It is also required in the sample chamber by the detectors commonly used to detect secondary electrons. The simplest solution is to share a common vacuum in the column and sample chamber. However, this approach imposes severe constraints on the sample. It must be clean, dry and non-volatile to avoid damage to the vacuum and electron optical systems. In addition, it must be conductive, in order to dissipate any charge that accumulates from interactions with the beam. Sample preparation procedures can extend the application range of high-vacuum SEM but impose a penalty in time, effort, and cost. Equally important, sample preparation can change the sample, interfering with accurate interpretation of both images and analytical results.

Low-Vacuum SEM - The limitations of high-vacuum SEM led to the

and transmitted electron detection, X-ray spectrometry, electron

backscatter diffraction, and focused ion beam (FIB) manipulation. The current generation of systems combine speed, flexibility, repeatability, and ease of use, making them the ideal solution for any laboratory that must satisfy a wide range of imaging and analytical demands.

\title{
Basics
}

All SEMs form an image by scan ning a finely focused beam of electrons over the sample surface in a raster pattern and collecting signals generated by interactions between the beam (primary) electrons and the sample atoms at each point in the pattern. The signal levels are displayed as variations in intensity in a two-dimensional image. Digital storage, manipulation and display technologies have almost completely replaced the analog displays and photographic techniques used in earlier SEM generations.
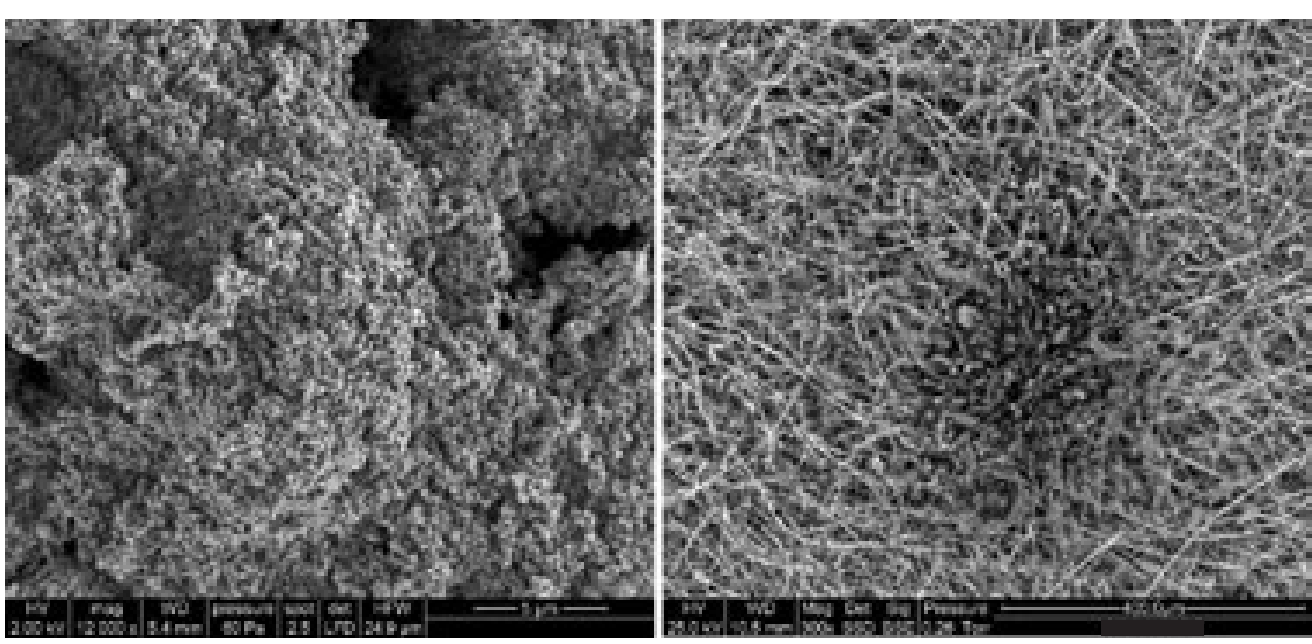

Fig 4 Uncoated PTFE particles - Uncoated PTFE charges heavily in high vacuum mode but the application of coatings can hide critical detail in the image. This secondary electron image was acquired in low vacuum mode using a special low vacuum detector.

Fig 5 Uncoated Filter-This low magnification backscattered electron image of an uncoated filter, acquired in low vacuum mode, has excellent image quality with no evidence of charging. 



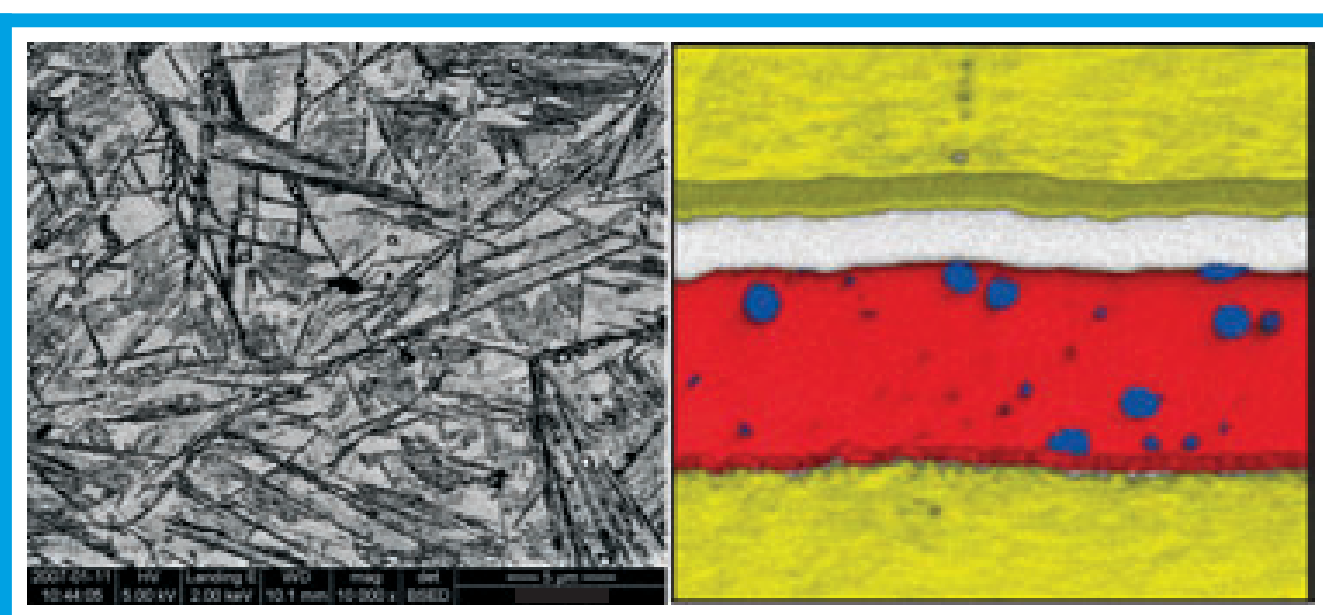

Fig 6 Phase distribution in etched steel. The atomic number contrast in this backscattered image clearly delineates the many phases present in the etched steel sample. The image was acquired in high vacuum mode using an advanced surface imaging technique that decelerates beam electrons just before they reach the sample to reduce penetration and enhance surface information. Maintaining higher energy throughout the column but reducing the landing energy of the beam optimizes electron optical performance and reduces the risk of damaging the sample.

Fig $7 X$-ray imaging - In this false colored X-ray image color indicates the location of various elements in the sample. The image demonstrates the excellent resolution available in STEM mode close to the sample, beyond the final optical aperture, to reduce the distance that the beam must travel through the higher pressure environment-the beam gas path length (BGPL). Shortening the BGPL reduces scattering of the primary beam.

ESEM technology provides secondary electron imaging with a detector specifically designed to operate in the extended vacuum regime. The detector, known as a gaseous secondary electron detector (GSED), actually uses gas in the sample chamber as part of the detection mechanism. An anode above the sample surface creates an electric field that accelerates low energy secondary electrons emerging from the sample surface toward the detector. The accelerated electrons collide with gas, creating positive ions and additional electrons. The ionization process can repeat many times, before the electrons reach the detector, resulting in a development of low-vacuum systems. Low-vacuum systems isolate the column vacuum from the sample chamber, usually by increasing the pumping capacity just above the final optical aperture. This pump

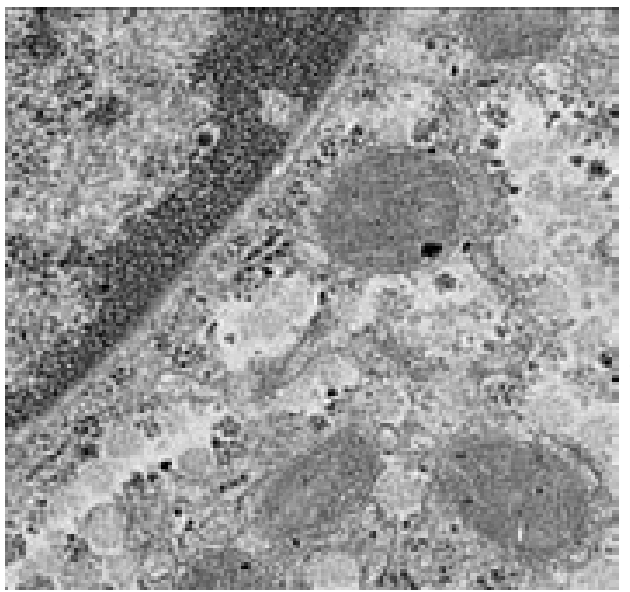

Fig 8 Liver cells-This STEM image shows the superb imaging resolution of STEM extracts most of the gas passing through the aperture before it can travel up the column. Although conventional (Everhardt Thornley) secondary electron (SE) detectors, which use high voltages to attract secondary electron, cannot function in the low vacuum conditions of the sample chamber, backscattered electron (BSE) detectors can. BSE images do not offer the resolution, topographic contrast and surface sensitivity of SE images, but BSE's higher energies make them less susceptible to charging effects. Low-vacuum systems of this type can operate at pressures up to a few hundred Pascals. For applications where BSE imaging is sufficient, they can eliminate much of the sample preparation required for high vacuum SEM. Secondary electron detectors that neutralize charge in the low vacuum range are also now available (see GSED discussion below).

ESEM - Extended low-vacuum SEM (ESEM, also known as Environmental SEM) was developed specifically to extend SEM capability to much higher pressures. Pressures above $600 \mathrm{~Pa}$ can sustain wet samples indefinitely without drying and provide a mechanism for effective charge neutralization. To achieve these pressures, ESEM uses one or more pressure limiting apertures (PLA) and a multistage differentially pumped vacuum system that isolates the column vacuum from the sample environment. The PLA can be positioned

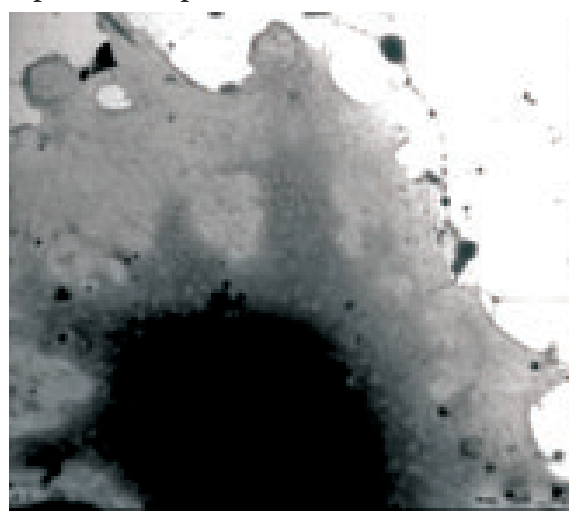
cascade amplification of the initial SE signal. A very important benefit of the gaseous secondary electron detection technology is its ability to prevent charging. The positive ions created by the cascade neutralize any charge accumulating on the sample and eliminate the need to apply conductive coatings to insulating samples.

ESEM extends the application of SEM to sample types and dynamic processes that are otherwise off limits. Examples include particles on wet filter substrates or suspended in liquid films, deformation of insulating materials, melting and crystallization of ceramics (at temperatures up to $1500^{\circ} \mathrm{C}$ ), temperature and humidity cycling of pharmaceuticals, and many more.

\section{Resolution, resolution, resolution}

What are the first three priorities of SEM? Resolution, resolution and resolution. Continual improvements in low vacuum technology, particularly ESEM, allows current generation systems to offer uncompromised performance across the full range of vacuum conditions. Resolution in any SEM is determined by the size spot formed by the beam at the sample surface, and, for small spots, by the size of the region within the sample from which the detected signal originates. ESEM technology addresses both of these factors. Shortening the BGPL reduces scattering of the primary beam that would otherwise broaden the beam, degrading both resolution and contrast. Close examination of the scattering process reveals that rather than generally broaden-
Fig 9 STEM imaging in the ESEM can be used on wet samples as well as dry. Bright field (left) and dark field (right) images of a mouse fibroblast show the expected contrast reversal. (Image courtesy Maarten Moes, University Utrecht)

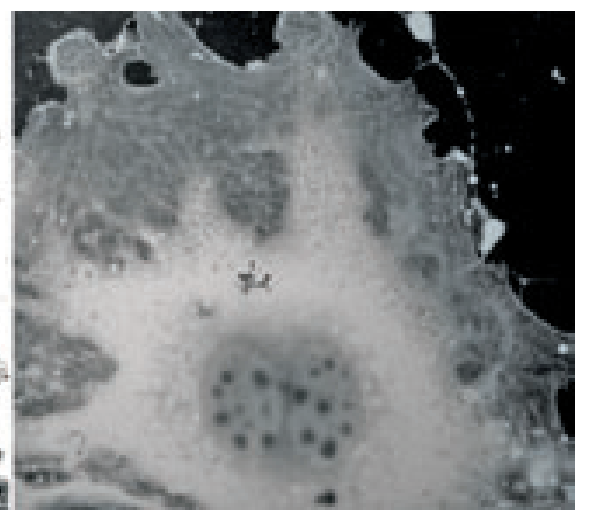
. 


\section{Beating the Competition is Easy, When You've Got Products They Don't Offer!}

Si-Li EDS Detectors, IXRF offers guaranteed, premium $\leq 130 \mathrm{eV}$, resolution Si-Li detectors. (*industry standard detector resolution $133-138 \mathrm{eV}$ ) that carry a three year warranty.

New $30 \mathrm{~mm}^{2}$ at $133 \mathrm{eV}$, three year warranty available for cost effective fast X-ray Mapping.

\section{fX SEM XRF,}

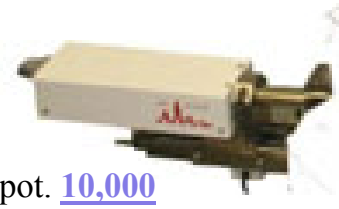

times more analytically sensitive than EDS.

Perfect for environmental applications as well as non-conductive samples.

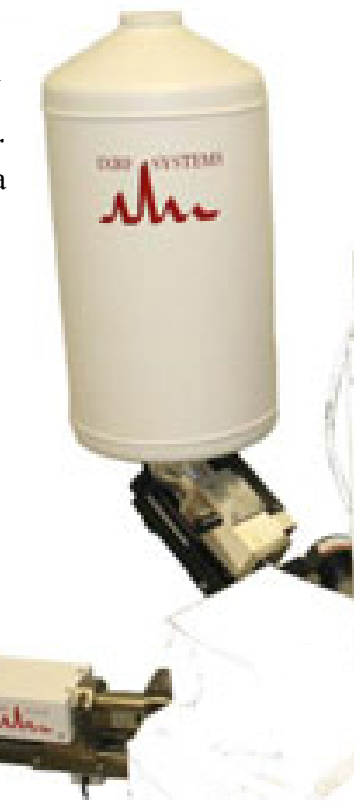

X-Beam, Micro XRF, 40-60 micron spot. 10,000 times more analytically sensitive than EDS. Out performs WDX for less than half the cost.

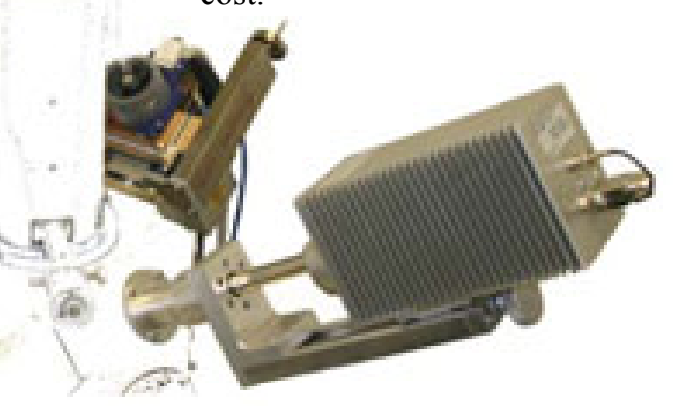

$50 \mathrm{~mm}^{2}$ Silicon Drift Detector, the largest Active Area in the industry $\left(50 \mathrm{~mm}^{2}\right)$. $133 \mathrm{eV}$ resolution, with optimum peak stability, and high input count rates. Who says "Bigger isn't better"?

IXRF does not stratify their software suite into low, medium, and high-end levels; there's only one high-end package that includes a myriad of Spectra Analysis, Image Analysis, X-ray

Mapping, and unsurpassed SEM/EDS Automation.

\section{Free Software Upgrades for Life...no compromises.}




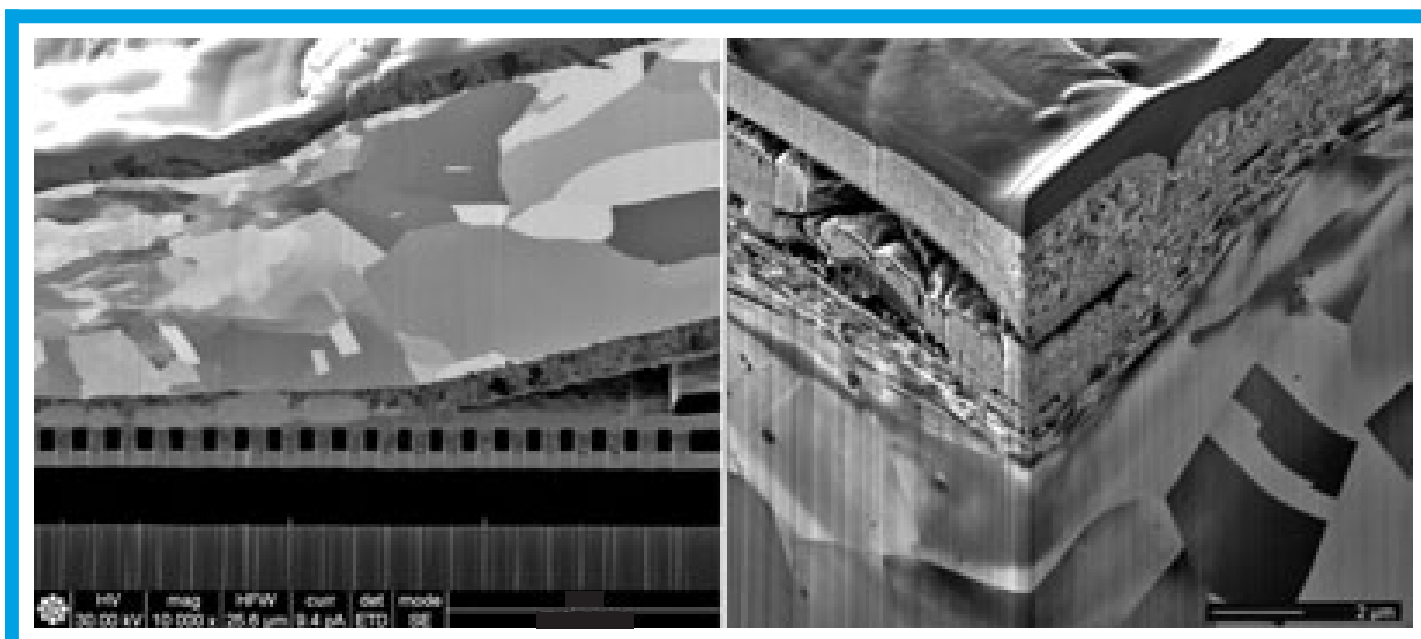

Fig 10 Gold coated bond wire. FIB is an imaging technique as well as a means of manipulating the sample. This image of a cross section through a gold coated wire bond was acquired using the FIB induced secondary electron signal. Channeling contrast clearly delineates the grain structure of the wire and its gold coating.

Fig 11 Corrosion and anti corrosion coatings on steel. FIB cross sectioning reveals subsurface detail in this FIB induced secondary electron image of corrosion and anti corrosion coatings on a steel substrate. ing the Gaussian distribution of electrons across the spot diameter, increasing scattering reduces the amplitude of the sharply focused unscattered distribution, which is gradually subsumed within a much broader background distribution of scattered electrons. Within the partial scattering regime most useful for high resolution imaging, a significant portion of beam electrons remain unscattered and the intense imaging signal they generate can be separated from the low level background by simple thresholding. Shortening the BGPL increases the ratio of scattered to unscattered electrons, retaining the small spot size and improving the dynamic range (contrast) of the thresholded signal. The cascade amplification of the gaseous secondary electron detector is a clean process, contributing negligible noise to the output signal. Finally, the secondary electron signal amplified by the GSED generally provides higher resolution than backscattered electrons-secondary electrons have low energies and can escape from the sample only if they are created very near the surface. As a result, most secondary electrons originate within or very close to the beam spot.

Advances in GSED design have extended its performance and operational range. Maximum gain and $\mathrm{S} / \mathrm{N}$ ratio are a function of BGPL, cascade path length, gas pressure and detector voltage. In its basic configuration the gas path lengths of the primary beam and the secondary electron cascade are roughly equivalent, creating a trade off between scatter in the primary beam and amplification gain in the secondary cascade. Decoupling the path lengths, by manipulating the physical configuration of the detector or the shape of local magnetic and electric fields, has allowed designers to extend GSED performance with specific capabilities such as operation in higher vacuum or with wider field of view.

Advances in detector design have also improved the spatial resolution of compositional maps obtained with
$\mathrm{X}$-ray spectrometry. Unlike the secondary electron signal, which is relatively strong and retains good resolution and contrast even when a threshold is applied to remove the component generated by scattered beam electrons, $\mathrm{X}$-ray count rates are relatively low. Thresholds are ineffective for reasonable acquisition times and typically every detected $\mathrm{X}$-ray is included in the count recorded for each pixel in the image array. With a beam path length of several millimeters even low angle scattering can land electrons many micrometers outside the beam spot. These "skirt" electrons can generate $\mathrm{X}$-rays that are not characteristic of the beam location. Once again, specially designed detectors that shorten the BGPL reduce the skirt diameter and improve the spatial resolution of X-ray maps.

\section{STEM}

Scanning transmission electron microscopy (STEM) generally offers much better imaging and analytical resolution than SEM. The size of the region within the sample from which the detected signal originates for a given beam position is the ultimate determinant of image resolution in any scanning technique. The size and shape of this region, known as the volume of interaction (VOI), is determined by the diameter of the beam spot, by the paths followed by primary electrons as they penetrate and scatter within the sample, and by the ability of the various signals to escape from the sample to be detected. The VOI can be quite large, (several microns) in bulk specimens for high beam energies and signals such as X-rays that travel freely through the sample. Thin samples, required by STEM to permit electron transmission, eliminate most of the volume in which scattering would occur in a bulk sample. The STEM beam has little opportunity to spread and the volume it interrogates corresponds closely to the region directly below the beam spot. The spatial resolution of images and analyses in STEM approaches the diameter of the beam.

Performing STEM in an SEM requires little more than positioning an appropriate detector below the sample. However, generating the small diameter beam necessary to realize the resolution benefits

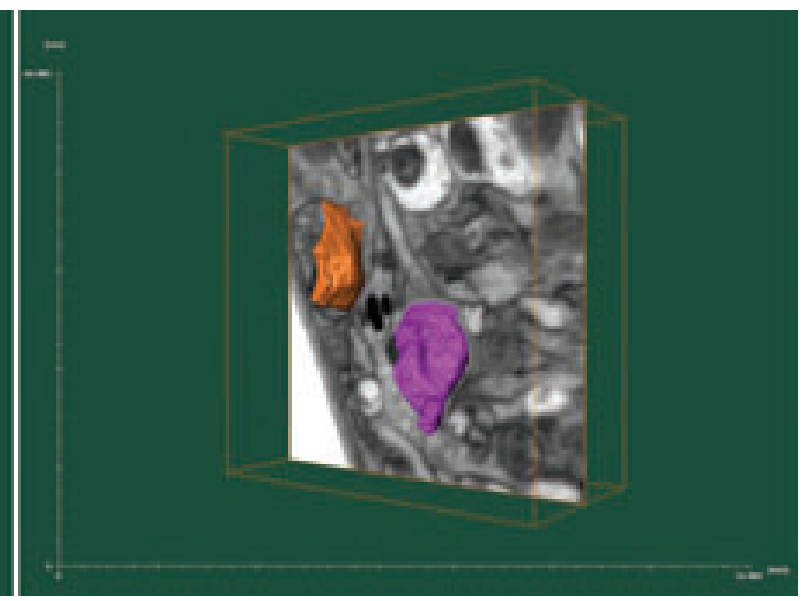

Fig 12 Mouse neural tissue. SlicedView automatically cuts and images a series of cross sections progressing through a region or feature of interest. Reconstruction software then assembles the image series into a three dimensional model that can be rotated, resectioned and false colored in the virtual model space. 


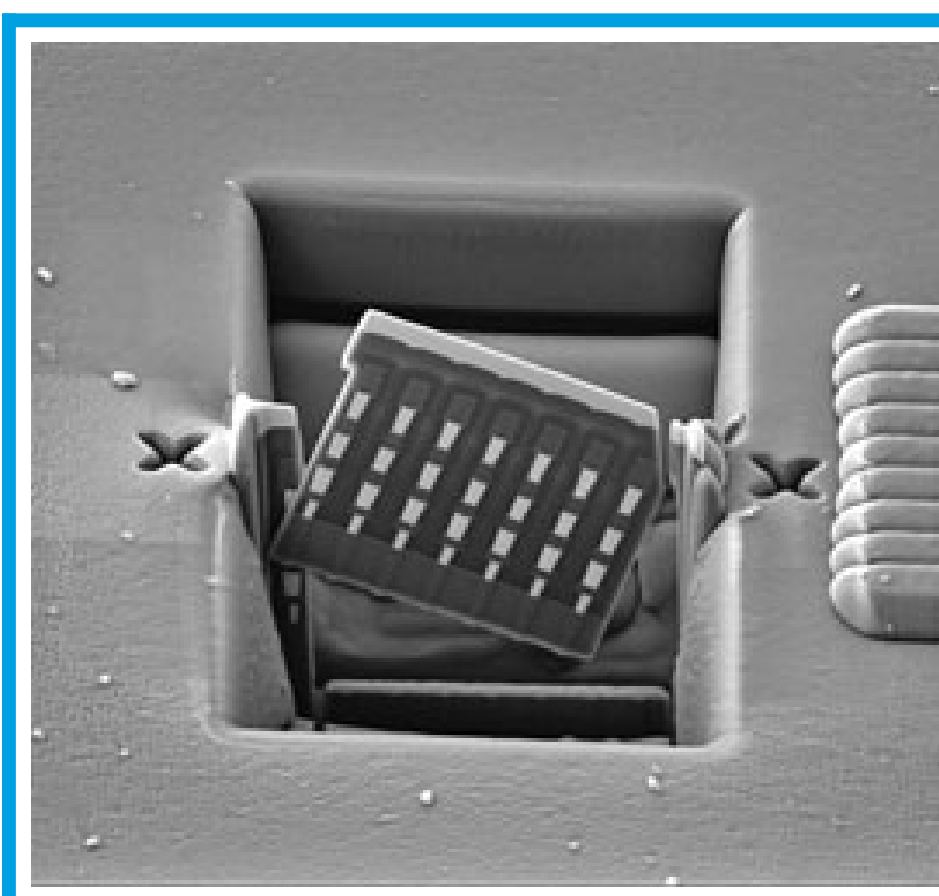

Fig 13 FIB prepared TEM sample. Automated, FIB based preparation can cut preparation time for thin STEM and TEM samples from days to hours. This thin section was created by milling material from both sides of the targeted feature. It has been cut free and is ready to be lifted out and transferred to a TEM grid.

of STEM in a low vacuum SEM can be problematic. The minimum diameter of a beam with usable current levels is determined largely by the electron source. Field emission sources provide spots nearly an order of magnitude smaller than tungsten sources at equivalent currents. $\mathrm{LaB}_{6}$ spot sizes fall in between. The problem for low vacuum SEM is that these smaller-spot sources also require increasingly higher vacuum levels. An SEM with a field emission source operating in the extended low vacuum range must maintain a vacuum differential between the sample chamber $(>1000 \mathrm{~Pa})$ and the gun $\left(<10^{-7} \mathrm{~Pa}\right)$ greater than ten orders of magnitude, while still providing a straight, unobstructed path for the electron beam - a non trivial exercise in vacuum design. However, it can be done. Advanced systems such as FEI's Quanta 3D FEG offer sub-nanometer resolution in STEM mode. Although the improvement in STEM imaging resolution is significant, the most dramatic benefits are in the spatial resolution of X-ray maps, which may improve from several microns to the near nanometer range. Historically, the difficulty of preparing the thin samples $(<100 \mathrm{~nm})$ required for STEM impeded its wide spread adoption. However, as the next section describes, the addition of a focused ion beam to the SEM provides a fast, easy solution for STEM (and TEM) sample preparation.

\section{Three Dimensional Dual Beam Imaging and Analysis}

DualBeam systems like FEI's Quanta 3D series combine the flexible imaging and analysis of capabilities of low vacuum SEM with the imaging and sample manipulation capabilities of a focused ion beam. FIB images offer unique benefits in certain applications, for example, their strong channeling contrast is useful in delineating the grain structure of polycrystalline materials. But it is the FIB's ability to add and remove material from the sample with nanoscale control that makes the dual beam such a versatile and powerful tool.

As noted above, FIB milling can be used to prepare the thin samples required for TEM and STEM. Demand for the high resolution capabilities of S/TEM has grown explosively in recent years, particularly in semiconductor manufacturing where device dimensions are shrinking beyond the resolving power of SEM. Manual preparation procedures are slow, difficult, and unreliable. Automated routines that use the FIB to excise a small section from the sample and thin that section to electron transparency can cut preparation time from several days to less than an hour. In a DualBeam, simultaneous SEM imaging of the FIB milling process permits precise control, allowing the operator to stop thinning when the desired features are revealed and the proper thickness is reached. The roughly uniform rate at which FIB mills different materials facilitates preparation of composite samples containing both hard and soft materials.

Surely one of the most valuable applications of the FIB's milling capability is its use to reveal subsurface structure for high resolution imaging with the electron beam. Electrons do not penetrate most materials very deeply, making SEM primarily a near surface technique. In some circumstances this is an advantage-low voltage SEM is exquisitely sensitive to the surface topography of the sample. In other cases, three dimensional information is required. In a DualBeam, the electron and ion beams intersect at the sample surface at an angle of about $52^{\circ}$ with the surface normal to the ion beam. In this configuration, the surface of a cross section cut by the FIB is immediately available for SEM imaging. As in STEM sample preparation, simultaneous imaging of the milling process permits fine control and insures the process is halted when the desired feature is exposed.

Charging can be a problem during FIB cross sectioning of insulating materials. The ion beam is composed of positive gallium ions and negative charge escapes from the sample as secondary electrons. Charging can distort or deflect the ion beam, degrading the precision of milling and deposition operations. In a DualBeam, simultaneous illumination of the cross section with a defocused, low voltage electron beam can prevent the accumulation of positive charge.

Recent advances in automation have taken 3D structural imaging to a new level. The Slice\&View technique automatically cuts and images a series of cross sections progressing through the feature of interest. Reconstruction software then assembles the sequence of images into a three dimensional model that can be rotated, resectioned, and otherwise manipulated in the virtual model space.

Both the ion and electron beams can be used to deposit material on the sample surface with the same nanoscale control as the FIB milling process. The material to be deposited, in the form of a volatile precursor compound, is introduced in the vicinity of the beam through a small needle. The beam decomposes the precursor molecules, depositing the non volatile component on the sample surface while the volatile components are pumped away by the vacuum system. Milling and deposition can be combined to build elaborate three dimensional nanoscale prototypes. The same type of injector used for material deposition can be used to inject gases that selectively increase FIB milling rates on specific materials. Gas assisted milling can be used to speed up a milling operation or to delineate material interfaces in a cross sectional image.

\section{Conclusion}

Current-generation low-vacuum SEMs and dual beams offer uncompromised performance in high vacuum mode and significantly extend the applicability of SEM. ESEM technology extends SEM to the widest range of sample types and chamber conditions and provides unique imaging and charge neutralization capabilities. Together, high vacuum, low vacuum and ESEM capabilities constitute the most versatile SEM solution available, increasing equipment utilization and allowing the microscopists to respond to almost any imaging or analytical situation. The elimination of most sample preparation requirements provides faster, more repeatable results while preserving the integrity of the sample. 